\title{
Adaptive laboratory evolution of $\beta$-caryophyllene producing Saccharomyces cerevisiae
}

\author{
Avinash Godara ${ }^{1}$ and Katy C. Kao ${ }^{1,2^{*}}$ (1)
}

\begin{abstract}
Background: $\beta$-Caryophyllene is a plant terpenoid with therapeutic and biofuel properties. Production of terpenoids through microbial cells is a potentially sustainable alternative for production. Adaptive laboratory evolution is a complementary technique to metabolic engineering for strain improvement, if the product-of-interest is coupled with growth. Here we use a combination of pathway engineering and adaptive laboratory evolution to improve the production of $\beta$-caryophyllene, an extracellular product, by leveraging the antioxidant potential of the compound.

Results: Using oxidative stress as selective pressure, we developed an adaptive laboratory evolution that worked to evolve an engineered $\beta$-caryophyllene producing yeast strain for improved production within a few generations. This strategy resulted in fourfold increase in production in isolated mutants. Further increasing the flux to $\beta$-caryophyllene in the best evolved mutant achieved a titer of $104.7 \pm 6.2 \mathrm{mg} / \mathrm{L}$ product. Genomic analysis revealed a gain-of-function mutation in the a-factor exporter STE6 was identified to be involved in significantly increased production, likely as a result of increased product export.
\end{abstract}

Conclusion: An optimized selection strategy based on oxidative stress was developed to improve the production of the extracellular product $\beta$-caryophyllene in an engineered yeast strain. Application of the selection strategy in adaptive laboratory evolution resulted in mutants with significantly increased production and identification of novel responsible mutations.

Keywords: Adaptive laboratory evolution, $\beta$-Caryophyllene, Selective pressure, Saccharomyces cerevisiae, CRISPRCas9

\section{Background}

Terpenoids are the largest and most diverse family of plant-derived compounds found in nature. They are high-value compounds with wide industrial applications ranging from fuel alternatives, nutraceuticals, pharmaceutics, etc. Terpenes are secondary metabolites that are derived from $\mathrm{C}_{5}$ precursor isopentenyl diphosphate (IPP) or dimethylallyl diphosphate (DMAPP) [1, 2]. Extracting

*Correspondence: katy.kao@sjsu.edu

${ }^{2}$ Department of Chemical and Materials Engineering, San Jose State University, One Washington Sq, San Jose, CA 95192, USA

Full list of author information is available at the end of the article them from their natural source is usually not economical because of their low abundance. Thus, microbial-based biosynthesis of terpenoids has been explored as a sustainable alternative for industrial production [3-5].

Sesquiterpene is a family of terpenoids containing three isoprene units and they can be monocyclic, bicyclic or tricyclic in structure $[6,7]$. $\beta$-caryophyllene is a bicyclic sesquiterpene and has antioxidant and anti-inflammatory properties, with potential applications as an aircraft fuel alternative and a potential therapeutic compound due to its antioxidant and anti-inflammatory properties $[8,9]$. Microbial production of $\beta$-caryophyllene has been demonstrated in E. coli and cyanobacteria reaching titers of 
$\sim 1.5 \mathrm{~g} / \mathrm{L}$ in a fed-batch bioreactor and $46.6 \mu \mathrm{g} / \mathrm{L}$, respectively $[10,11]$.

Apart from rationally engineering the known terpenoid biosynthesis pathway, due to complex and interlinked nature of metabolic network and cellular physiology, genes and pathways not directly connected to the biosynthetic pathway may influence product formation [12, 13]. A clear knowledge of the genotype-phenotype relationship for product formation is not fully known. Thus, complementary techniques to rational engineering such as screening deletion or overexpression libraries [14-16] and adaptive laboratory evolution (ALE)-based strategies $[17,18]$ can help to identify additional gene and/or pathway targets related with increasing product formation. In recent work, Promdonkoy et al. utilized both rational engineering and ALE to improve the D-xylose utilization and isobutanol production in Saccharomyces cerevisiae [19]. In another work, Rugbjerg et al. showed evolved Escherichia coli MG1655 which utilizes glucose more efficiently due to a mutation in $r p o B$ also exhibited improved mevalonate productivity [20].

Previously, we developed an environmental engineering-based strategy using ALE to improve product formation of an intracellular product with antioxidant properties by applying periodic oxidative stress challenge [18]. With extracellular products, the same strategy may fail due to potential "cheating" by non-producers in the population. In this work, we explore the potential application of ALE for the production of an extracellular product $\beta$-caryophyllene in S. cerevisiae. Initial pathway engineering led to a strain producing $\sim 3.8 \mathrm{mg} / \mathrm{g}$ DCW $\beta$-caryophyllene. The optimized strain was used to design an optimum oxidative stress challenge strategy to improve product formation using ALE. Using the optimized strategy, evolved mutants that exhibit a fourfold increase in $\beta$-caryophyllene biosynthesis were isolated and characterized. Subsequent genotypic analyses led to the identification of two beneficial mutations responsible for the enhanced production phenotype observed.

\section{Results and discussion}

\section{Optimization of oxidative stress for use in adaptive laboratory evolution}

Selecting an appropriate selective pressure (stressor) is key to successfully use of adaptive laboratory evolution. Since $\beta$-caryophyllene is a known antioxidant $[9$, 21], an oxidative stress-based selective pressure can be used to aid the coupling of cellular growth or survival with production [18]. The strategy is based on the hypothesis that in an oxidative environment, the strain that produces more of an antioxidant product will have a growth advantage. To determine whether the production of $\beta$-caryophyllene increased cell survival, the
QHS1 gene encoding $\beta$-caryophyllene synthase from Artemisia annua, which has been successfully used to biosynthesize $\beta$-caryophyllene in E. coli [11] was codon optimized for yeast and expressed in strain BY4741. The catalase gene encoded by CTT1 was deleted to reduce the yeast native defense against hydrogen peroxide. Then strains that produce varying levels of $\beta$-caryophyllene were constructed in the $\operatorname{ctt} 1 \Delta$ strain, resulting in non-producers YAG110 (BY4741 ctt1D) and YAG114 (YAG110 with a genome-integrated farnesyl pyrophosphate [FPP] overproduction cassette), and producers YAG111 (YAG110 with the $\beta$-caryophyllene synthase QHS1 gene integrated in the genome) and YAG115 (YAG110 with QHS1 and FPP overproduction cassette integrated in the genome). See Additional file 1: Figure S1 and "Materials and methods" section for description of genes used in metabolic engineering. Initial experiment was conducted to measure the level of $\beta$-caryophyllene accumulated intracellularly and in the culture. No $\beta$-caryophyllene was detected from the intracellular measurements (data not shown), suggesting that this is an extracellular product. Production results showed YAG115 producing higher amount of $\beta$-caryophyllene than YAG111 (Additional file 1: Table S1). All four strains were challenged with different concentrations of $\mathrm{H}_{2} \mathrm{O}_{2}(0 \mathrm{mM}, 50 \mathrm{mM}, 100 \mathrm{mM}$, $150 \mathrm{mM}$ and $200 \mathrm{mM}$ ) and their relative viabilities were assessed (results are shown in Additional file 1: Figure S2). The non-producers YAG110 and YAG114 showed the lowest levels of tolerance with $\sim 10 \%$ survival with $50 \mathrm{mM} \mathrm{H}_{2} \mathrm{O}_{2}$. Strain YAG111 showed an intermediate level of oxidative stress tolerance with $\sim 10 \%$ survival after challenge with $150 \mathrm{mM} \mathrm{H}_{2} \mathrm{O}_{2}$. The strain with the highest level of $\beta$-caryophyllene production YAG115 exhibited the highest tolerance with $\sim 10 \%$ survival at $200 \mathrm{mM} \mathrm{H}_{2} \mathrm{O}_{2}$.

In addition to survival after $30 \mathrm{~min}$ challenge with higher concentrations of $\mathrm{H}_{2} \mathrm{O}_{2}$, cellular growth during continuous exposure at growth-permissible concentrations were also evaluated for each strain. $\mathrm{H}_{2} \mathrm{O}_{2}$ concentrations ranging from $0 \mathrm{mM}, 25 \mathrm{mM}, 50 \mathrm{mM}, 75 \mathrm{mM}$ and $100 \mathrm{mM}$ were directly added to the media, and growth kinetics were measured. The data (in Additional file 1: Table S2) showed no growth in the non-producer YAG110 for any $\mathrm{H}_{2} \mathrm{O}_{2}$ concentrations above $0 \mathrm{mM}$. Strain YAG111 and YAG114 showed some growth in $100 \mathrm{mM}$ and $50 \mathrm{mM}$ respectively. The higher tolerance of YAG114 over YAG110 may be attributed to increased accumulation of sterol because of the presence of the FPP overproduction cassette. The highest producer, YAG115, was the only strain able to grow in $100 \mathrm{mM}$ hydrogen peroxide, although with a very long lag phase and with a low final cell density. This data showed the possibility of using 
continuous exposure as a possible alternative to periodic challenge as environmental stress for ALE experiment.

YAG115 was chosen as the parental strain for ALE to improve $\beta$-caryophyllene production since this strain exhibited the highest tolerance to oxidative stress. Prior to initiating an evolution experiment, two types of oxidative stress selection strategies were evaluated for their ability to improve $\beta$-caryophyllene production. A continuous exposure strategy, where the cultures are continuously exposed to growth-permissible $\mathrm{H}_{2} \mathrm{O}_{2}$ in the media, and a periodic exposure strategy, where the cultures are subjected to a $30 \mathrm{~min} \mathrm{H}_{2} \mathrm{O}_{2}$ challenge followed by a recovery period as described in Fig. 1, were used. In all cases, a dodecane layer $(500 \mu \mathrm{L})$ was added to each $3 \mathrm{~mL}$ of culture for $\beta$-caryophyllene capture. After every $24 \mathrm{~h}$ of growth, $\sim 7 \%(200 \mu \mathrm{L})$ of the culture was used to inoculate the subsequent culture (3 $\mathrm{mL}$ of fresh media). Each strategy was evaluated for $\beta$-caryophyllene titer and yield after 8 days (continuous strategy) or 4 cycles (periodic challenge strategy). Results showed no significant increase in yield using continuous exposure at $25 \mathrm{mM} \mathrm{H}_{2} \mathrm{O}_{2}$ compared to the no stressor control (Fig. 2). A decrease in total yield was observed for all other $\mathrm{H}_{2} \mathrm{O}_{2}$ concentrations using continuous exposure. On the other hand, significant increases of up to 1.7 -fold in production were observed in populations subjected to periodic challenge with $50 \mathrm{mM} \mathrm{H} \mathrm{O}_{2}$. Periodic challenge with $100 \mathrm{mM} \mathrm{H}_{2} \mathrm{O}_{2}$ resulted in an insignificant increase in production, with higher concentrations showing complete loss of production. The results revealed that periodic challenge at a $\mathrm{H}_{2} \mathrm{O}_{2}$ concentration that resulted in $\sim 10 \%$ survival was an optimal selection strategy for increasing $\beta$-caryophyllene production using ALE.

\section{Increased $\beta$-caryophyllene production via adaptive laboratory evolution using periodic $\mathrm{H}_{\mathbf{2}} \mathrm{O}_{\mathbf{2}}$ challenge}

An ALE experiment was initiated with YAG115 using the periodic challenge strategy with an initial concentration of $50 \mathrm{mM} \mathrm{H}_{2} \mathrm{O}_{2}$. To further optimize the rampup in the ALE, at the end of cycle 2 (day 5), the culture was split into two populations, $\mathrm{P} 1$ and $\mathrm{P} 2$, which were exposed to $50 \mathrm{mM}$ and $100 \mathrm{mM} \mathrm{H}_{2} \mathrm{O}_{2}$ exposure, respectively, in ramping up the selection pressure (Additional file 1: Figure S3). The next split and ramp-up was done at the end of cycle 4 (day 9) from the P2 population (100 mM exposure) into P3 and P4, which were subsequently exposed to $150 \mathrm{mM}$ and $200 \mathrm{mM} \mathrm{H}_{2} \mathrm{O}_{2}$ exposure, respectively. After the split, four populations (P1, P2, P3, and P4) were maintained. At cycle 5, P1 and P2 reached a peak in production, reaching $\sim$ threefold increase in $\beta$-caryophyllene production before a stagnation or decrease in production were observed. On the other hand, populations P3 and P4 showed consistent decreases in production over time. Thus, population P2 was chosen for further analyses.
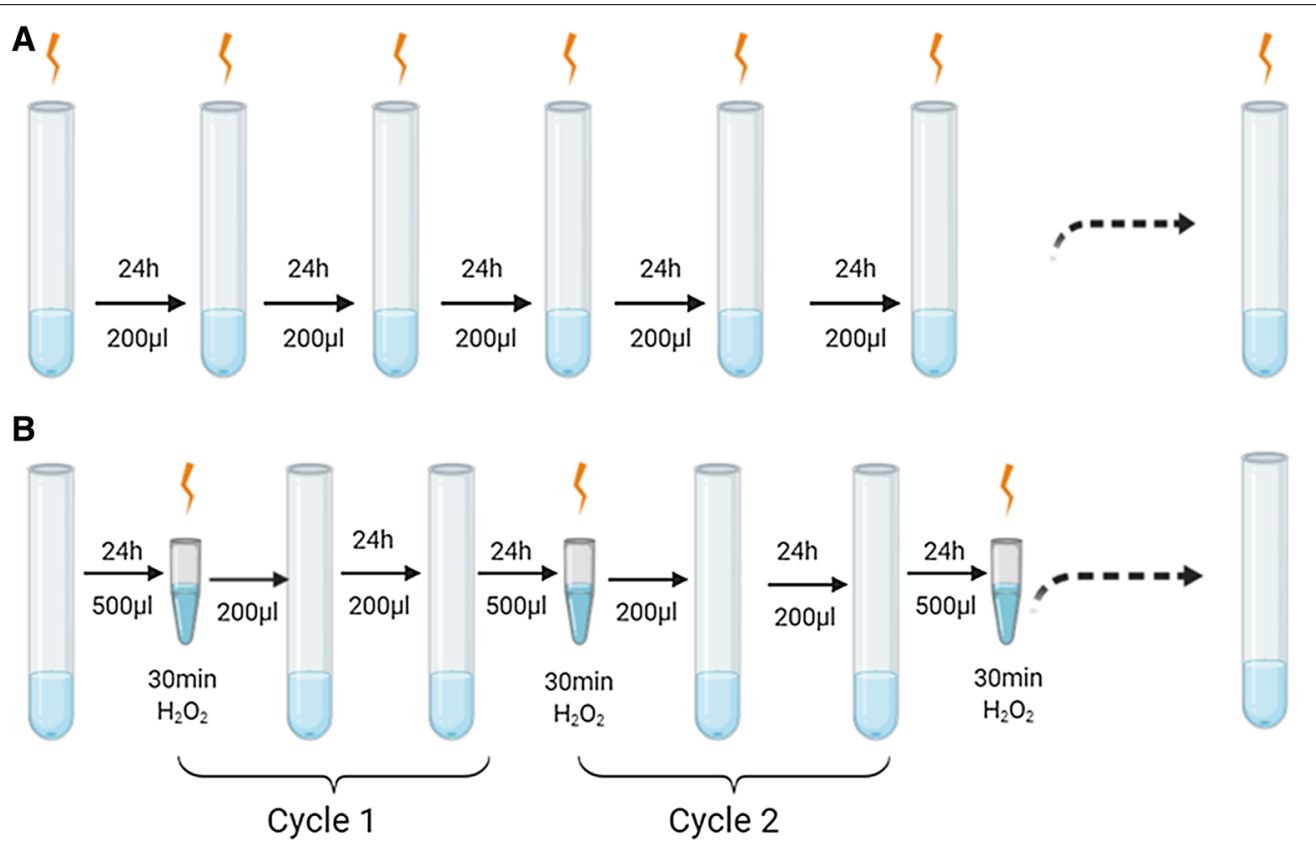

Fig. 1 Schematic of different stress strategy tested. A Continuous exposure with $\mathrm{H}_{2} \mathrm{O}_{2}$ directly added to media. B Periodic exposure to $\mathrm{H}_{2} \mathrm{O}_{2}$ for $30 \mathrm{~min}$, followed by a recovery period 

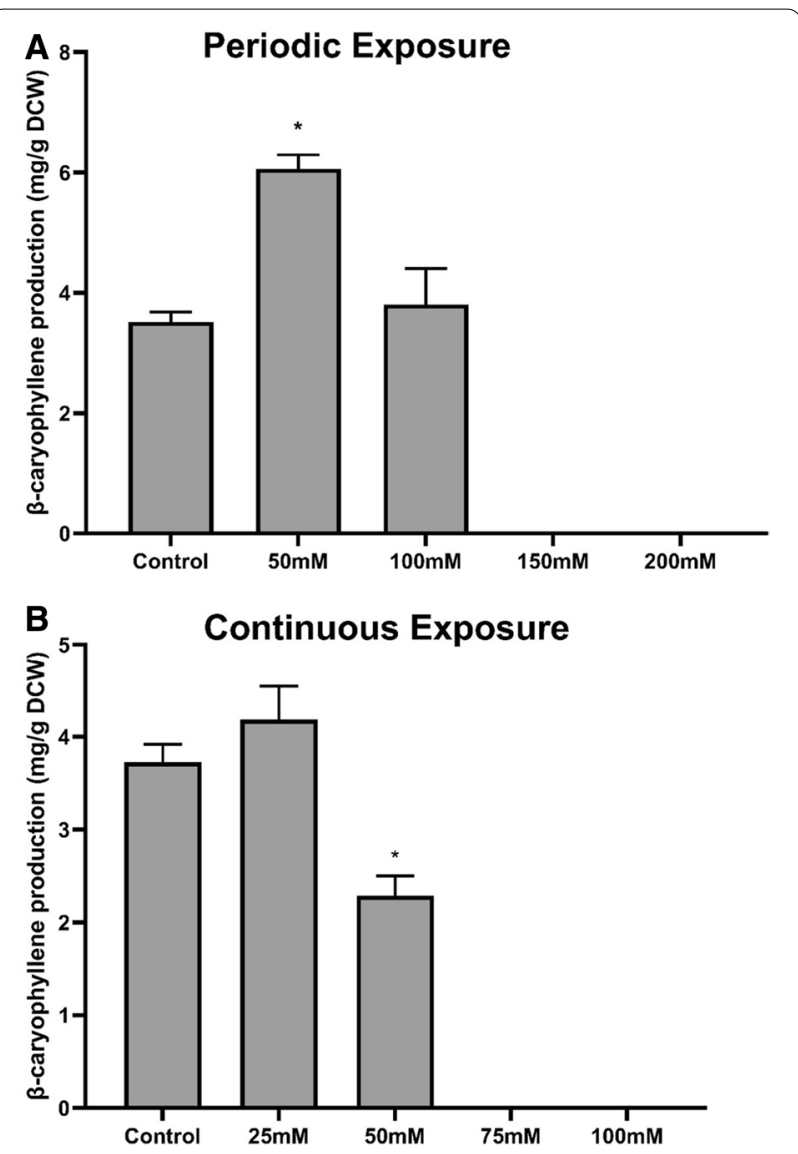

Fig. 2 Average $\beta$-caryophyllene production observed after short-term selection using hydrogen peroxide for 8 days using $\mathbf{A}$ periodic challenge and $\mathbf{B}$ continuous exposure at various hydrogen peroxide concentrations. Production was measured in $20 \mathrm{~mL}$ test tube with $3 \mathrm{~mL}$ culture overlayed with $500 \mu \mathrm{L}$ dodecane. Asterisks: $\mathrm{p}$ value $<0.05$ using two-tailed Student's t-test against control

\section{Isolated evolved mutants exhibit significant increase in production}

Under the assumption that evolved mutants with higher production of $\beta$-caryophyllene should have enhanced survival after oxidative stress challenge compared to the parental strain, we first identified a concentration of $\mathrm{H}_{2} \mathrm{O}_{2}$ in which the parental strain has negligible viability after a 30-min exposure. As shown in Additional file 1: Figure S4, $1 \mathrm{M} \mathrm{H}_{2} \mathrm{O}_{2}$ exposure resulted in no growth of the parental strain, whereas evolved population P2 (day 11 ) had $\sim 10 \%$ viability. In addition, we hypothesized that colony size after $\mathrm{H}_{2} \mathrm{O}_{2}$ challenge can be used to estimate relative production. Population P2 (day 11) was exposed to $1 \mathrm{M}$ hydrogen peroxide stress for $30 \mathrm{~min}$ and plated on SC-ura-leu plate. Three sizes of colonies were observed, small, medium and large. These colonies were cultured and their $\beta$-caryophyllene production were quantified. A positive correlation was observed in size and production as medium and large colonies (see Additional file 1: Figure S5). The result suggests a growth advantage of mutants with higher production of $\beta$-caryophyllene, indicating the evolution strategy resulted in growth/survival and production coupling as hypothesized.

Using a 30-min exposure to $1 \mathrm{M}$ hydrogen peroxide, we subjected four time point samples from the evolved population: P1 (day 4), P2 (day 8), P2 (day 11), P2 (day 14) and P2 (day 18) to screen for hyperproducers. We failed to identify any viable isolates from population P1 (day 4). For the remaining populations, eight individual colonies per population were randomly chosen. The isolated mutants were subjected to a second round of screening based on their $\beta$-caryophyllene production. Figure 3A shows the individual production obtained from each isolate. The top five overproducing evolved mutants were selected from the 32 total mutants, and their $\beta$-caryophyllene production were characterized in more detail. Results showed a two to fourfold increase in production compared to the parental strain, with mutant P11M1 (population 2 day 11 mutant 1) being the best performer as shown in Fig. 3B. The stability of P11M1 was assessed via four serial passages in SC-ura-leu media (3 days of growth each passage for a total of 12 days); $\beta$-caryophyllene production was monitored after each passage. The parental strain was included as control. P11M1 showed consistent production of $\sim 16 \mathrm{mg} / \mathrm{g}$ DCW, whereas the control produced $\sim 4 \mathrm{mg} / \mathrm{g} \mathrm{DCW}$.

To determine whether QHS1 is the rate-limiting step in $\beta$-caryophyllene production in the evolved mutants, the QHS1 gene was overexpressed using $2 \mu$ plasmid into both the parental and the best evolved isolate P11M1, resulting in strain YAG116 and YAG117, respectively. Overexpression of QHS1 led to modest increases in production in the parental strain from $\sim 4 \mathrm{mg} / \mathrm{g} D C W$ $(20.1 \pm 0.3 \mathrm{mg} / \mathrm{L})$ to $\sim 5.3 \mathrm{mg} / \mathrm{g} \mathrm{DCW}(27.8 \pm 2.1 \mathrm{mg} / \mathrm{L})$ and P11M1 from $\sim 16(89.7 \pm 2.4 \mathrm{mg} / \mathrm{L})$ to $\sim 18 \mathrm{mg} / \mathrm{g}$ DCW (104.7 $\pm 6.2 \mathrm{mg} / \mathrm{L})$ (Fig. 4), suggesting that $\beta$-caryophyllene synthase is not a major rate-limiting step in $\beta$-caryophyllene production in the evolved mutants.

\section{Genome sequencing identified two beneficial mutations for $\beta$-caryophyllene production}

To identify beneficial mutations for the observed increased production in the isolated mutants, we sequenced four time-course population samples from population P2 (day 8 , day 11 , day 14 and day 18), five of best isolated mutants, and the parental strain. A mutation frequency cutoff of greater than or equal to 0.8 was used to narrow down the dominant mutations in the population. Large numbers $(>200)$ of mutations above the cutoff threshold were observed in the population samples. The identified mutations in single 

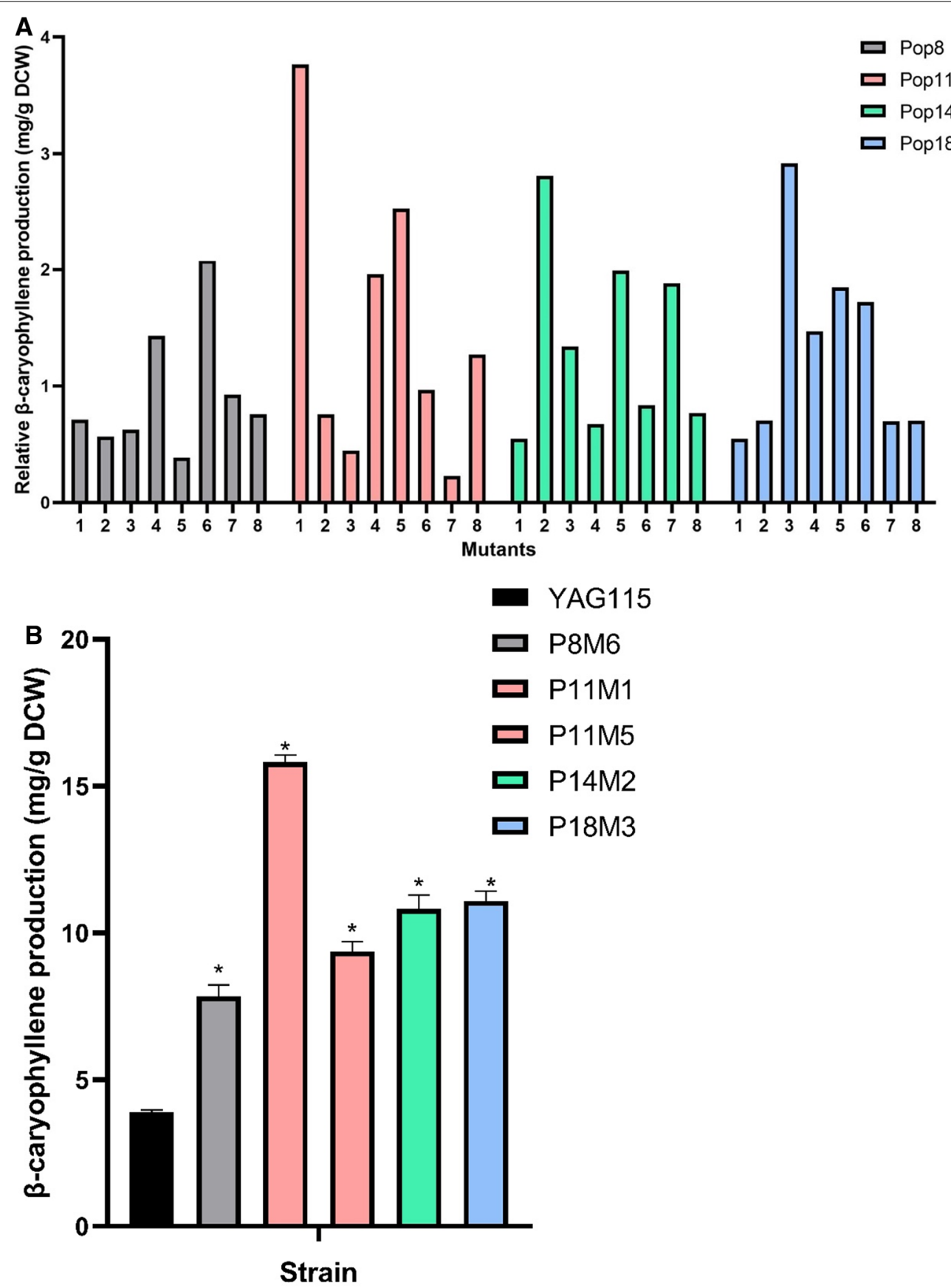

Fig. 3 Isolated evolved mutants exhibited increased $\beta$-caryophyllene production over the parental strain. A Initial screening of isolated mutants from each evolved population based on relative product yield in 48 well plates. Pop8 = P2 (day 8), Pop $11=$ P2 (day 11), Pop 14=P2 (day 14), and Pop18 = P2 (day 18). Production was measured in 48 well plate with $1 \mathrm{~mL}$ culture overlayed with $166 \mu \mathrm{L}$ dodecane. B Confirmation of product formation in the five best performing mutants. Production was measured in $20 \mathrm{~mL}$ test tube with $3 \mathrm{~mL}$ culture overlayed with $500 \mu \mathrm{L}$ dodecane. The mutants are named first by the population they were isolated from followed by the isolate number. For example, P11M1 is the first mutant from Pop11. Asterisks: $p$ value $<0.05$ using two-tailed Student's t-test against control

hyperproducer isolates are shown in Additional file 1: Table S3. The high frequency mutations (in populations) that were also identified in sequenced single isolates from the respective populations were selected for further analyses. In addition, since isolate P11M1 was the best producer identified, all mutations in P11M1 were 


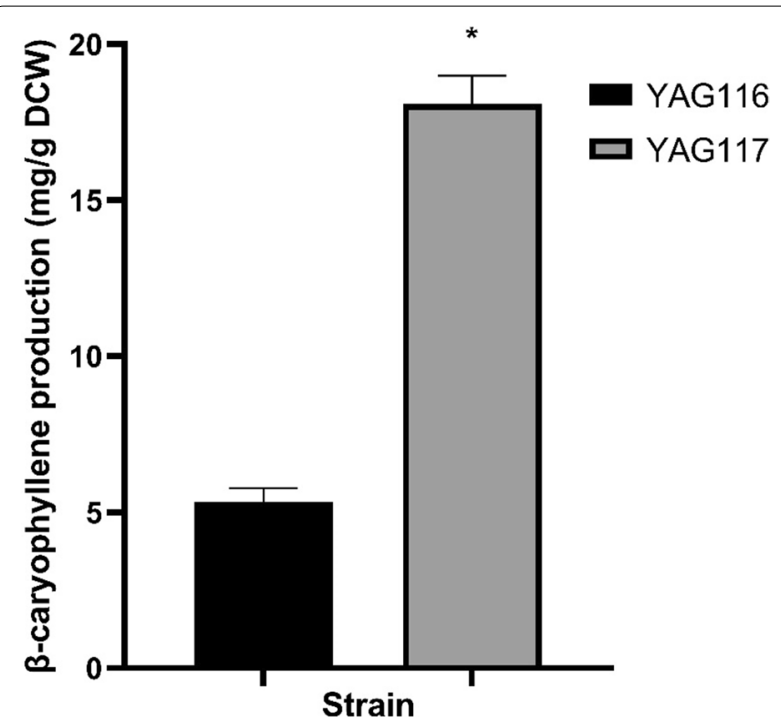

Fig. 4 Production of $\beta$-caryophyllene in strains with additional copies of QHS1 gene. Production was measured in $20 \mathrm{~mL}$ test tube with $3 \mathrm{~mL}$ culture overlayed with $500 \mu \mathrm{L}$ dodecane. Asterisks: $p$ value $<0.05$ using two-tailed Student's t-test compared with YAG1 16

also chosen, for a total of 13 unique mutations selected for further analyses (Table 1). To determine the impacts of each of these mutations on $\beta$-caryophyllene biosynthesis, single mutations were introduced into parental strain using site-directed mutagenesis, resulting in strains YAG132-145. Only two mutations led to improved production, the mutation in STE6 gene and a mutation in intergenic region of between MST27/tR(UCU)G1 (Fig. 5 and Additional file 1: Table S4). STE6 T1025N mutation improved production 3.7 fold to $12.6 \mathrm{mg} / \mathrm{g} \mathrm{DCW}$ of $\beta$-caryophyllene. The MST27/tR(UCU)G1 intergenic mutation improved production threefold to $10.3 \mathrm{mg} / \mathrm{g}$ DCW of $\beta$-caryophyllene compared to the parental strain. No further increase in production was observed when these two mutations were combined.

\section{Growth kinetics and oxidative tolerance of reconstructed mutations}

In order to investigate the general fitness benefit conferred by the individual mutations identified, we did a growth kinetics study for all individual reconstructed strains. The data showed that five $(C D C 39, t K(C U U) C /$ MAK32 int, RIF1 D709N, STE6 T1025N and YAL064W $B / T D A 8$ int) out of 13 mutations did not impact growth under normal culture conditions (see Table 2). The FLO9 T288T, RNR3/FIS1 int, and SNO2/SNZ2 int mutations exhibited increased growth rates. The DAN4 T200T, MDS3, MTL1 S257S, MST27/tR(UCU)G1 int, and FLO9 T288T mutations showed significant reduction in lag phase.

To assess the impacts of these mutations on oxidative stress tolerance, the individual reconstructed mutants ( $\beta$-caryophyllene producers) were subjected to $200 \mathrm{mM}$ hydrogen peroxide exposure for $30 \mathrm{~min}$. The same set of mutations (listed in Table 1) were also reconstructed in a background strain lacking the $\beta$-caryophyllene synthase gene, resulting in strains YAG119-YAG131, which were used to study the impacts of these mutations on oxidative stress tolerance in the absence of production. In the absence of $\beta$-caryophyllene production, an increased 10X survival compared to the reference strain (YAG114) was observed in YAG121 (FLO9 T288T), YAG123 (MDS3), YAG124 (MST27/tR(UCU)G1 int) and YAG125 (MTL1 $S 257 S$ ) (Table 3). In the $\beta$-caryophyllene producing background, $10 \mathrm{X}$ increased tolerance was observed in

Table 1 Mutations chosen for detailed characterization

\begin{tabular}{|c|c|c|c|c|c|}
\hline Chromosome & Position & Mutation & Amino acid change & Gene & Abbreviation \\
\hline 3 & 286312 & $\mathrm{C} \rightarrow \mathrm{T}$ & Nonsense mutation & CDC39 & CDC39 \\
\hline 10 & 715141 & $A \rightarrow G$ & T200T & DAN4 & DAN4 T200T \\
\hline 1 & 27105 & $A \rightarrow G$ & T288T & FLO9 & FLO9 T288T \\
\hline 3 & 151555 & $+\mathrm{A}$ & Intergenic & tK(CUU)C/MAK32 & tK(CUU)C/MAK32 int \\
\hline 7 & 128474 & $\mathrm{~T} \rightarrow \mathrm{A}$ & Nonsense mutation & MDS3 & MDS3 \\
\hline 7 & 404475 & $+G$ & Intergenic & MST27/tR(UCU)G1 & MST27/tR(UCU)G1 int \\
\hline 7 & 530034 & $A \rightarrow C$ & S257S & MTL1 & MTL1 S257S \\
\hline 2 & 754982 & $\mathrm{C} \rightarrow \mathrm{T}$ & D709N & RIF1 & RIF1 D709N \\
\hline 9 & 241053 & $(\mathrm{~A})_{21 \rightarrow 22}$ & Intergenic & RNR3/FIS1 & RNR3/FIS1 int \\
\hline 14 & 12986 & $(\mathrm{~T})_{11 \rightarrow 14}$ & Intergenic & SNO2/SNZ2 & SNO2/SNZ2 int \\
\hline 11 & 43222 & $\mathrm{G} \rightarrow \mathrm{T}$ & $\mathrm{T} 1025 \mathrm{~N}$ & STE6 & STE6 T1025N \\
\hline 1 & 12690 & $\mathrm{~A} \rightarrow \mathrm{T}$ & Intergenic & YAL064W-B/TDA8 & YAL064W-B/TDA8 int \\
\hline 8 & 2303 & $(C)_{11 \rightarrow 12}$ & Intergenic & YHL050C/YHL050C & YHL050C int \\
\hline
\end{tabular}




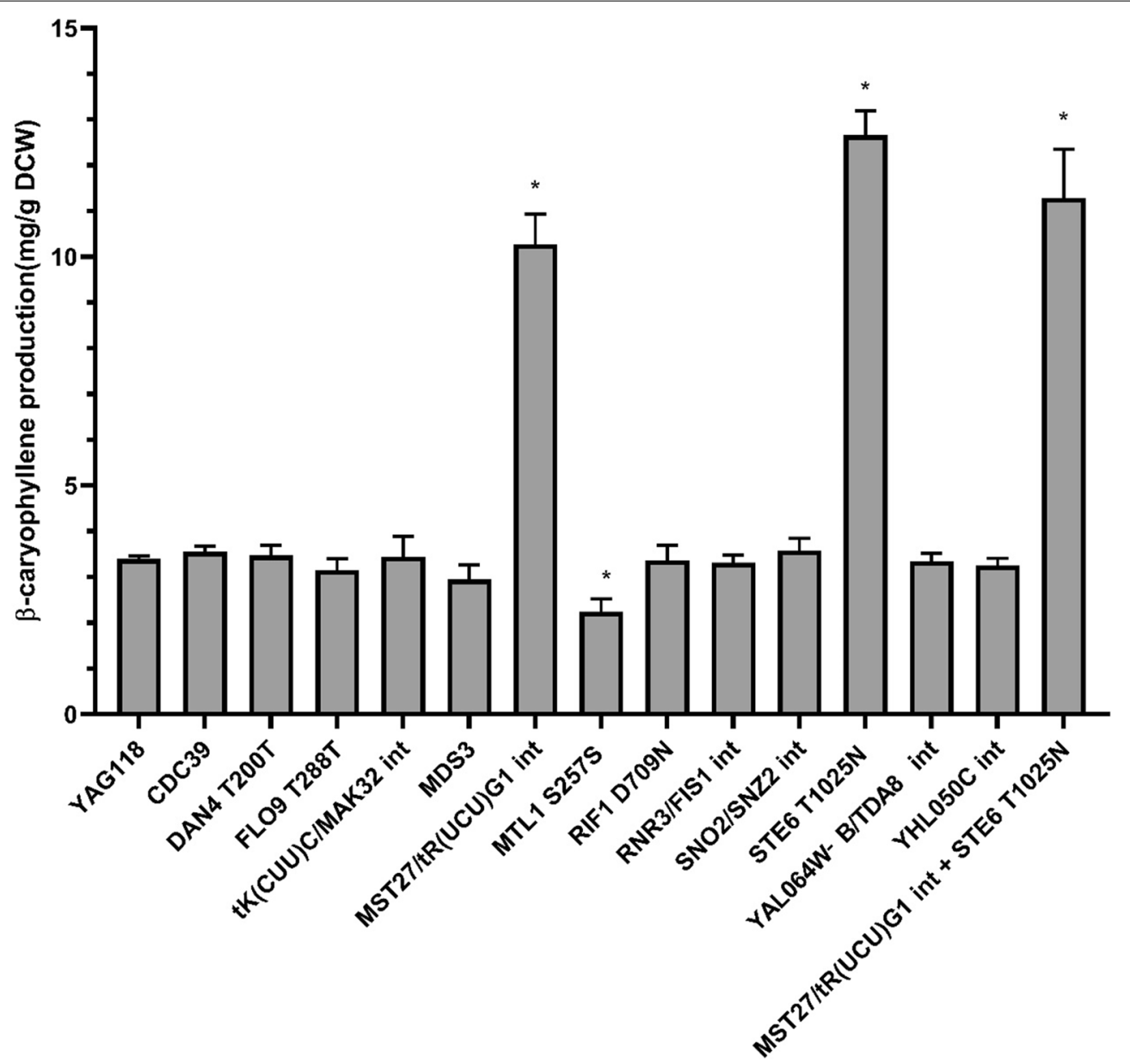

Fig. 5 Production of $\beta$-caryophyllene in reconstructed single mutants. Production was measured in $20 \mathrm{~mL}$ test tube with $3 \mathrm{~mL}$ culture overlayed with $500 \mu \mathrm{L}$ dodecane. Asterisks: $p$-value $<0.05$ using two-tailed Student's t-test against control

YAG134 (FLO9 T288T), YAG136 (MDS3), YAG137 (MST27/tR(UCU)G1 int), YAG138 (MTL1 S257S) and YAG142 (STE6 T1025N).

\section{Effect of STE6 T1025N on caryophyllene production}

Since the missense mutation in STE6 led to a large impact on $\beta$-caryophyllene production, we assessed whether the mutation is a loss-of-function or gain-of-function mutation using STE6 knockout (YAG146) and overexpression (YAG147 with STE6 T1025N and YAG148 with wild-type STE6) strains, and quantifying their effects on $\beta$-caryophyllene production (data shown in Fig. 6). The STE6 knockout strain exhibited no change in production compared with the reference. Production was also not impacted when the wild type STE6 was overexpressed. However, when we overexpressed the mutated STE6 in the reference strain, $\beta$-caryophyllene production increased fourfold to $13.8 \mathrm{mg} / \mathrm{g} \mathrm{DCW}$, suggesting the STE6 T1025N mutation is a gain-of-function mutation.
We further explored the possibility that the STE6 $T 1025 \mathrm{~N}$ mutation may influence the efflux and thus the production of other sesquiterpenes by overexpressing the mutated gene in an engineered $\alpha$-humulene producer. No increase in production was observed in the strain that overexpresses STE6 T1025N compared to the reference (Additional file 1: Figure S6), suggesting that the mutation specifically affects the transport of $\beta$-caryophyllene and does not significantly influence the efflux of sesquiterpenes in general.

\section{Discussion}

$\beta$-Caryophyllene was produced in yeast using heterologous QHS1 gene and production was further improved using overexpression of intermediate FPP. Periodic (30 $\mathrm{min}$ ) and continuous exposure to hydrogen peroxide at various concentrations demonstrated the benefit of $\beta$-caryophyllene production on oxidative stress protection. Leveraging this oxidative stress protection of 
Table 2 Growth kinetics data for reconstructed strains

\begin{tabular}{|c|c|c|c|c|c|c|c|}
\hline Strain & Mutations & $\begin{array}{l}\text { Growth rate } \\
\left(\mathrm{h}^{-1}\right)\end{array}$ & Stdev & Lag phase (h) & Stdev & OD600Max & Stdev \\
\hline YAG118 & Control & 0.17 & 0.01 & 3.35 & 0.14 & 1.05 & 0.02 \\
\hline YAG132 & CDC39 & 0.18 & 0.01 & 3.34 & 0.27 & 1.09 & 0.06 \\
\hline YAG133 & DAN4T200T & 0.18 & 0.02 & 2.71 & 0.28 & 1.04 & 0.05 \\
\hline YAG134 & FLO9T288T & 0.20 & 0.01 & 2.19 & 0.38 & 1.15 & 0.01 \\
\hline YAG135 & tK(CUU)C/MAK32 int & 0.16 & 0.02 & 3.43 & 0.15 & 0.99 & 0.09 \\
\hline YAG136 & MDS3 & 0.18 & 0.01 & 0.72 & 0.14 & 1.07 & 0.04 \\
\hline YAG137 & MST27/tR(UCU)G1 int & 0.17 & 0.01 & 2.39 & 0.10 & 1.06 & 0.04 \\
\hline YAG138 & MTL1 S257S & 0.18 & 0.01 & 1.12 & 0.15 & 1.05 & 0.05 \\
\hline YAG139 & RIF1 D709N & 0.19 & 0.01 & 3.37 & 0.11 & 1.09 & 0.02 \\
\hline YAG140 & RNR3/FIS1 int & 0.20 & 0.01 & 3.20 & 0.10 & 1.12 & 0.06 \\
\hline YAG141 & SNO2/SNZ2 int & 0.20 & 0.01 & 3.21 & 0.02 & 1.18 & 0.02 \\
\hline YAG142 & STE6 T1025N & 0.18 & 0.01 & 3.15 & 0.23 & 1.08 & 0.08 \\
\hline YAG143 & YAL064W-B/TDA8 int & 0.17 & 0.03 & 3.18 & 0.35 & 1.10 & 0.14 \\
\hline YAG144 & YHL050C int & 0.17 & 0.01 & 3.68 & 0.03 & 1.07 & 0.06 \\
\hline P11M1 & Multiple & 0.19 & 0.01 & 2.34 & 0.18 & 1.11 & 0.05 \\
\hline
\end{tabular}

Bold: $p$ value $<0.05$ using two-tailed Student's t-test compared with control

Table 3 Hydrogen peroxide stress tolerance for strains with and without $\beta$-caryophyllene production after $200 \mathrm{mM} \mathrm{H}_{2} \mathrm{O}_{2}$ exposure for $30 \mathrm{~min}$

\begin{tabular}{|c|c|c|c|c|c|}
\hline \multirow[t]{2}{*}{ Mutation } & \multicolumn{2}{|c|}{ No production } & \multicolumn{3}{|c|}{ With production } \\
\hline & Strain & $\begin{array}{l}\text { Relative } \mathrm{H}_{2} \mathrm{O}_{2} \\
\text { tolerance }\end{array}$ & Strain & $\begin{array}{l}\text { Relative } \mathrm{H}_{2} \mathrm{O}_{2} \\
\text { tolerance }\end{array}$ & $\begin{array}{l}\beta \text {-caryophyllene } \\
\text { (mg/g DCW) }\end{array}$ \\
\hline Control & YAG114 & & YAG118 & & 3.39 \\
\hline CDC39 & YAG119 & ND & YAG132 & ND & 3.55 \\
\hline DAN4T200T & YAG120 & ND & YAG133 & ND & 3.47 \\
\hline FLO9T288T & YAG121 & + & YAG134 & + & 3.15 \\
\hline $\mathrm{tK}(\mathrm{CUU}) \mathrm{C} / \mathrm{MAK} 32 \mathrm{int}$ & YAG122 & ND & YAG135 & ND & 3.44 \\
\hline MDS3 & YAG123 & + & YAG136 & + & 2.94 \\
\hline MST27/tR(UCU)G1 int & YAG124 & + & YAG137 & + & 10.27 \\
\hline MTL1 S257S & YAG125 & + & YAG138 & + & 2.24 \\
\hline RIF1 D709N & YAG126 & ND & YAG139 & ND & 3.36 \\
\hline RNR3/FIS1 int & YAG127 & ND & YAG140 & ND & 3.31 \\
\hline SNO2/SNZ2 int & YAG128 & ND & YAG141 & ND & 3.57 \\
\hline STE6 T1025N & YAG129 & ND & YAG142 & + & 12.66 \\
\hline YAL064W-B/TDA8 int & YAG130 & ND & YAG143 & ND & 3.35 \\
\hline YHL050C int & YAG131 & ND & YAG144 & ND & 3.25 \\
\hline
\end{tabular}

Production was measured in $20 \mathrm{~mL}$ test tube with $3 \mathrm{~mL}$ culture overlayed with $500 \mu \mathrm{L}$ dodecane. ND: not different from wild-type. +: $~ 10 \times$ higher survival compared to wild-type. Bold: $p$ value $<0.05$ using two-tailed Student's t test compared with control

$\beta$-caryophyllene, two schemes of selective pressure were evaluated for 8 days for improving production. Continuous exposure resulted in no significant increase in production whereas periodic exposure resulted in 1.7-fold increment in production. Periodic challenge strategy was used for ALE experiment and resulted in threefold increment in production. Isolated mutants showed a correlation between size and production suggesting a growth advantage of mutants with higher production of $\beta$-caryophyllene.

Thirteen unique mutations were found through nextgen sequencing in population samples and isolated mutants. STE6 T1025N and intergenic mutation between $M S T 27 / t R(U C U) G 1$ showed the highest production 


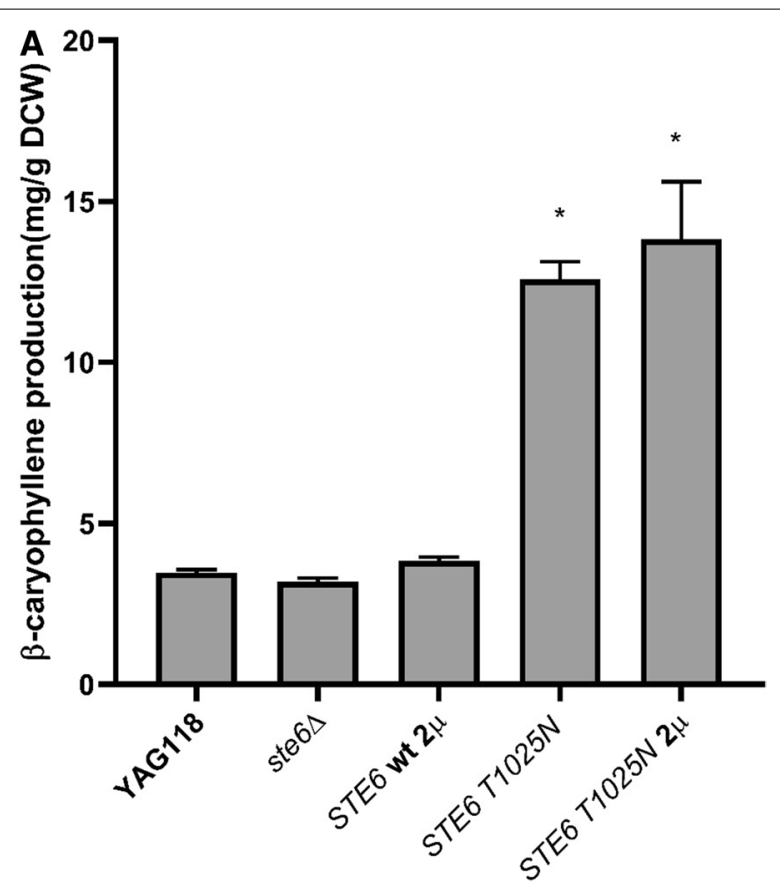

Fig. 6 The effect of STE6 expression on $\beta$-caryophyllene production. ste6 $\triangle$ : ste6 deletion strain. STE6 wt $2 \mu$ : wild-type STE6 expressed on a $2 \mu$ plasmid. STE6 T1025N: reconstructed parental strain with STE6 T1025N mutation. STE6 T1025N 2 $\mu$ : parental strain expressing mutated STE6 gene on a $2 \mu$ plasmid. Production was measured in $20 \mathrm{~mL}$ test tube with $3 \mathrm{~mL}$ culture overlayed with $500 \mu \mathrm{L}$ dodecane. Asterisk: $\mathrm{p}$ value $<0.05$ using two-tailed Student's t-test against parental strain

improvement. STE6 encodes the plasma membrane ATP binding cassette $(\mathrm{ABC})$ transporter known to export a-factor in MATa cells [22], and is a homolog to the mammalian multidrug resistance $m d r$ gene [23]. Bacteria commonly acquire mutations in efflux pumps to overcome stress conditions while producing terpenoids $[24,25]$. The STE6 T1025N mutation is a missense mutation, resulting in a change in amino acid from threonine to asparagine. A mutation in STE6 K1093A, which lies within a nucleotide binding domain, has previously been reported to increase the a-factor export in STE6 [26]. The STE T1025N mutation identified here is not located in any of the predicted nucleotide binding domains, and is 62 amino acids upstream of a conserved Walker A motif $[27,28]$. Since the mutation is located between the transmembrane and ATP binding domains on the predicted cytoplasmic portion of the protein, it potentially impacted substrate recognition, allowing increased export of $\beta$-caryophyllene. However, further experiments are needed to identify the exact effect the mutation has on transport of $\beta$-caryophyllene. The MST27/tR(UCU) G1 int mutation is an insertion mutation between MST27 and a Ty element [29]. MST27 is member of the DUP240 multi-gene family and known to impact the vesicles formation [30]. Ty1 elements is a transposon element and mutations in transposon elements are commonly found in evolution experiments and are known to impact fitness [31, 32]. However, as this mutation is in the intergenic region and at the $3^{\prime}$ end of $M S T 27$, it is difficult to ascertain the exact impact of the mutation on the cell.

Interestingly, both the STE6 T1025N and MST27/ $t R(U C U) G 1$ intergenic mutations were found in very low frequencies in the population (below detection limit). The STE6 T1025N mutation was found only in mutant P11M1 and MST27/tR(UCU)G1 intergenic mutation was identified in all the isolated mutants sequenced. Note that the population samples sequenced were cultured in normal growth conditions before harvesting for next-gen sequencing, while the isolated mutants underwent a round of selection in $1 \mathrm{M}$ hydrogen peroxide. The findings that the MST27/tR(UCU)G1 intergenic mutation was not detected in the population samples, but is present in all isolated mutants, and the positive correlation between cellular survival (based on colony size after $\mathrm{H}_{2} \mathrm{O}_{2}$ challenge; Additional file 1: Figure S5) and product formation, suggest that the evolving population was highly heterogeneous with the most productive members consisting of a smaller fraction of the population.

Mutation in FLO9 T288T positively impacted specific growth rate, lag phase, and max OD600, suggesting a significant fitness benefit, which likely allowed it to reach fixation in the evolving population. The synonymous mutation in FLO9 changed the codon for threonine from ACT to the less preferred ACC, which may impact translation of the protein. Mutations and changes in copy number in the $F L O$ genes have been found to be involved in tolerance to environmental stressors [33-35]. Mutations in all FLO9 and MDS3 were found in detectable frequencies in all populations sequenced, and the MTL1 mutation was present at $~ 49 \%$ frequency in the P11 population, suggesting that the increased oxidative stress tolerance conferred by these mutations likely contributed to their selection in the ALE experiment. The MTL1 S257S mutation is also a synonymous mutation, with a serine codon change from TCA to a less preferred TCC, suggesting this mutation may lead to reduced translation of the protein. Mutations in FLO9 and MTL1 were found in an industrial yeast strain that was evolved for growth on hydrolysates inhibitors [33], which have been shown to induce oxidative stress in $S$. cerevisiae [36]. The increased hydrogen peroxide tolerance in YAG142 (STE6 T1025N) can be attributed to increased production, as without $\beta$-caryophyllene production there was no benefit to survival in oxidative stress challenge. The intergenic mutation in $M S T 27 / t R(U C U) G 1$, which is one of the two mutations found to be responsible for increased $\beta$-caryophyllene production, showed general 
fitness benefit with reduced lag phase in normal growth conditions and $\sim 10 \times$ increase in survival in the presence of hydrogen peroxide stress. While this mutation is likely selected for due to the fitness it confers in the presence of strong oxidative stress, although it's frequency in the population is extremely low, it is unclear how it contributed to increased $\beta$-caryophyllene production.

\section{Conclusions}

In this work, we explored the use of adaptive laboratory evolution to improve the production of an extracellular product, $\beta$-caryophyllene, using oxidative stress challenge as selection. Initial metabolic engineering by overexpressing isoprenoid pathway genes resulted in a parental strain that produces $3-4 \mathrm{mg} / \mathrm{g}$ DCW of $\beta$-caryophyllene. An ALE strategy was optimized by comparing periodic stress challenge versus continuous exposure. Using the optimized periodic $\mathrm{H}_{2} \mathrm{O}_{2}$ challenge method (from 50 to $200 \mathrm{mM} \mathrm{H}_{2} \mathrm{O}_{2}$ ), the ALE experiment resulted in evolving populations with up to $15.8 \mathrm{mg} / \mathrm{g} \mathrm{DCW}$ (>threefold increase) in $\beta$-caryophyllene production. Under the assumption that a positive correlation exists between oxidative stress tolerance and $\beta$-caryophyllene production, a final selection using $1 \mathrm{M} \mathrm{H}_{2} \mathrm{O}_{2}$ for 30 min was used to isolate mutants with the highest level of oxidative stress tolerance. This strategy yielded isolated mutants with up to fourfold increase in $\beta$-caryophyllene production. Mutations in the intergenic region of MST27/tR(UCU)G1 and a non-synonymous mutation in STE6 were the only mutations found to benefit product formation. STE6 is a known $\mathrm{ABC}$ exporter for a-factor in yeast. Deletion and overexpression studies demonstrated that the mutation is a gain-of-function mutation. It may be possible that the STE6 T1025N mutation resulted in binding and export of $\beta$-caryophyllene. However, the exact mechanism for how this point mutation is affecting $\beta$-caryophyllene production is still unclear. Overall, we demonstrated that by leveraging the antioxidant potential of terpenes that are exported extracellularly, product formation can be coupled with cellular growth/survival and be rapidly improved using short-term ALE experiments.

\section{Materials and methods}

\section{Strains, plasmids and growth conditions}

All yeast strains used in this work are derivatives of S288c and listed in Additional file 1: Table S5. S. cerevisiae strain BY4741 (MATa, his3 $\Delta 1$, leu2 $\Delta 0$, met $15 \Delta 0$, ura3 $\Delta 0$ ) [37] are used as the base strains in this study. Yeast strains are cultured in Synthetic Complete (SC) media lacking specific amino acids for selection at $30^{\circ} \mathrm{C}$. E. coli strains used for subcloning were cultured at $37^{\circ} \mathrm{C}$ in Luria Broth (LB) supplemented with appropriate antibiotics. Cytosolic catalase T (CTT1) gene was deleted in BY4741 strain as described in our earlier work [18]. For terpene quantification, yeast strains were cultured in YPD at $30{ }^{\circ} \mathrm{C}$ and $200 \mathrm{rpm}$ for $72 \mathrm{~h}$.

\section{Plasmid construction for QHS1 and FPP overproduction}

The heterologous genes QHS1 from Artemisia annua [11] were codon optimized for S. cerevisiae and synthesized (Integrated DNA Technologies). Codon optimized QHS1 gene was integrated into the yeast genome or expressed on plasmid under $U R A 3$ selection marker. To increase flux towards FPP, $t H M G 1, H M G 2(K 6 R), U P C 2-1$ and $E R G 20$ were PCR amplified from yeast genome and added to chromosome at LEU2 locus under LEU2 selection. The truncated HMG1, tHMG1, contains the1575-bp C-terminal part of $H M G 1$ and was amplified from yeast genome using PCR and a start codon was added in the forward primer. The plasmids were constructed using the MoClo-YTK plasmid kit [38] (Addgene). In brief, each gene was introduced into the entry vector YTK001 using BsmBI (Thermo Scientific) via Golden Gate assembly protocol described by [38]. Each gene (in an entry vector) is then assembled into transcriptional unit plasmid along with the appropriate promoter and terminator part vectors using BsaI (Thermo Scientific) via Golden Gate assembly. Finally, different transcriptional units are joined together to be used as plasmid or genomic integration with different selection markers for yeast. DH5 $\alpha$ chemically competent $E$. coli cells were used for cloning and were prepared using Zymo Mix \& Go! E. coli Transformation Kit and Buffer Set ${ }^{\mathrm{TM}}$ (Zymo Research). Yeast competent cells were prepared using Frozen-EZ Yeast Toolkit II Kit ${ }^{\mathrm{TM}}$ (Zymo Research). To produce $\alpha$-humulene ZSS1 from Zingiber zerumbet was codon optimized for S. cerevisiae and synthesized (Integrated DNA Technologies) and introduced into genome as described above. pTDH3 was used as promoter and tTDH1 was used as terminator for ZSS1.

\section{Quantification of $\beta$-caryophyllene production}

Organic layer of dodecane on top of media was used to capture volatile $\beta$-caryophyllene produced by strains [39]. Unless specified a ratio of six-part media and onepart dodecane was used. A volume of $100 \mu \mathrm{L}$ of the dodecane overlay was sampled after 3 days or $24 \mathrm{~h}$ (for evolution experiment) for use in product quantification. Quantitative analysis of $\beta$-caryophyllene in dodecane layer was performed using gas chromatography [40]. Alpha-humulene was used as internal standard for peak normalization. An Agilent J\&W HP-5 (5\%-phenyl)methylpolysiloxane nonpolar column $(30 \mathrm{~m} \times 0.32 \mathrm{~mm}$ with $0.25 \mu \mathrm{m}$ film thickness) was used for this study. Gas chromatogram oven temperature was programmed from $100{ }^{\circ} \mathrm{C}$ initial temperature to $140{ }^{\circ} \mathrm{C}$ at $10{ }^{\circ} \mathrm{C} / \mathrm{min}$ rate, 
followed by $2.5{ }^{\circ} \mathrm{C} / \mathrm{min}$ to $180{ }^{\circ} \mathrm{C}$, followed by $20^{\circ} \mathrm{C} / \mathrm{min}$ until final temperature of $200{ }^{\circ} \mathrm{C}$. FID detector was kept at $280{ }^{\circ} \mathrm{C}$ whereas inlet was kept at $240{ }^{\circ} \mathrm{C}$ in a split-less mode. Flow was kept at $2 \mathrm{~mL} / \mathrm{min}$ with hydrogen flow at $30 \mathrm{~mL} / \mathrm{min}$ and ultra-pure air at $400 \mathrm{~mL} / \mathrm{min}$. Nitrogen was used for makeup flow at $25 \mathrm{~mL} / \mathrm{min}$. At least three biological replicates per strain were used for analysis.

\section{Growth kinetics measurements}

Microplate reader (TECAN Infinite ${ }^{\circledR} \mathrm{M}$ Nano) was used to measure growth curves for strains. Cells were grown for $24 \mathrm{~h}$ in test tubes, then normalized to $\mathrm{OD}_{600} \sim 0.05$ in $200 \mu \mathrm{L}$ final volume in media in 96-well plates. Cells were cultured in the microplate reader for $72 \mathrm{~h}$ with orbital shaking at constant intervals using kinetic cycles of 2 min incubation time, then orbital shaking for $3 \mathrm{~min}$ at $198 \mathrm{rpm}$ (3 mm amplitude) followed by OD600 measurements. To obtain specific growth rate $\mu$, duration of growth lag, and the maximum OD600, software grofit v1.1.1 [41] was used. Input file for grofit was generated and following script was ran in $\mathrm{R}$ :

growthdata <-read.csv("inputfile.csv",sep=",", header $=$ TRUE, check.names $=$ FALSE)

timedata <-read.csv("timeworksheet.csv", $\quad$ sep=",", header $=$ TRUE, check.names $=$ FALSE)

gro $<$-grofit(timedata, growthdata)

summary_table <- summary.gcFit(gro\$gcFit)

write.csv(summary_table,"Summary.csv")

When prompted to choose the models out of four preexisting model (logistic, richards, gompertz, or gompertz. exp), the models which fit best the growth curve was selected. Results were compiled from the summary table obtained from the software. Three biological replicates per strain were used for analysis.

\section{Oxidative stress tolerance using hydrogen peroxide}

For spot assays, overnight cultures were normalized to OD 600 of 1.0 using phosphate-buffered saline (PBS). Strains were treated with hydrogen peroxide for $30 \mathrm{~min}$ intervals in a shaking incubator at $30^{\circ} \mathrm{C}$ at $200 \mathrm{rpm}$. Cells were washed two times with PBS to eliminate the remaining hydrogen peroxide in solution. After the shock treatment, tenfold serial dilutions were performed in (PBS) and spotted on SC plates lacking appropriate amino acid. Three biological replicates per strain were used for analysis. For stress optimization and evolution experiment, the normalization step was omitted from the protocol.

\section{Adaptive laboratory evolution}

Single colonies of YAG115 were used to initiate the evolution experiment in $3 \mathrm{~mL}$ SC-ura-leu media on day 0 . For populations that were evolved using the constant exposure strategy, $200 \mu \mathrm{L}$ of cells were inoculated in fresh media supplemented with specified concentrations of $\mathrm{H}_{2} \mathrm{O}_{2}$. For populations that were subjected to periodic challenge, on day $1,500 \mu \mathrm{L}$ of culture was centrifuged, resuspended in phosphate buffer saline (PBS) and subjected to $50 \mathrm{mM} \mathrm{H}_{2} \mathrm{O}_{2}$ challenge for 30 min under shaking (challenge period) and washed twice with PBS after exposure. Population samples were preserved in glycerol stocks each day. $200 \mu \mathrm{L}$ of challenged cells were inoculated in $3 \mathrm{~mL}$ of SC-ura-leu media and $500 \mu \mathrm{L}$ of dodecane was added for $\beta$-caryophyllene capture. On day 2 , the population was allowed to recover by transferring $200 \mu \mathrm{L}$ of overnight culture into fresh media (recovery period). The populations were challenged on odd days with specified concentration of $\mathrm{H}_{2} \mathrm{O}_{2}$ and allowed to recover on even days. Production was quantified at the end of 24-h by recovering the dodecane layer for populations evolved using either the continuous or periodic challenge strategies.

\section{Isolating mutants from evolved populations}

To select for hyperproducing mutants from the evolved populations, each population was subjected to exposure to a higher concentration of hydrogen peroxide than that used during the ALE experiments. $500 \mu \mathrm{L}$ of each population sample was centrifuged and cells were resuspended in PBS with final concentration of $1 \mathrm{M}$ of hydrogen peroxide. After $30 \mathrm{~min}$ of exposure, the cells were washed 2 time with PBS, and all cells were plated on SC-ura-leu plates. Plates were kept for 2 days at $30{ }^{\circ} \mathrm{C}$. 8 colonies were randomly picked from each challenged population and streaked on SC-ura-leu plates to ensure we obtain individual clones. Single colonies were picked and cultured in 48 well plates containing $1 \mathrm{~mL}$ of media and $\sim 166 \mu \mathrm{L}$ dodecane overlay at $30{ }^{\circ} \mathrm{C}$ and shaking at $200 \mathrm{rpm}$. The dodecane layer was recovered to quantify $\beta$-caryophyllene production after $72 \mathrm{~h}$. To confirm the 48 well culture results, the high performing clones were grown in $3 \mathrm{~mL}$ cultures in test tubes with $500 \mu \mathrm{L}$ dodecane layer using three biological replicates.

\section{Whole genome resequencing}

Genomic DNA of isolated mutants, parental strain and population samples were extracted using YeaStar DNA extraction kit (Zymo Research). Library preparations and NGS sequencing were performed by the Texas A\&M Genomics Center for sequencing on the Illumina MiSeq platform using $300 \times 300$ paired-end reads using Nexterra DNAFlex kit for library generation. An average coverage of $>20$-fold was obtained for each isolated mutant and $>150$-fold for population samples. The sequencing data was aligned to S. cerevisiae S288c reference genome with breseq v0.29 [42]. De novo mutations in isolated mutants were identified by comparing against 
the YAG113 parental strain, and verified via Sanger sequencing. The raw sequencing data were deposited in SRA database (https://www.ncbi.nlm.nih.gov/sra) with accession number PRJNA669136.

\section{Reconstructing mutations into YAG 114 and YAG118 strain} CRISPR-Cas9 using the one plasmid-system with pCRCT developed by [43] was used for site-directed mutagenesis to reconstruct identified mutations into YAG118. Briefly, 120 bp sequence including donor sequence and guide sequence (Additional file 1: Table S6) was chemically synthesized by Twist Bioscience, USA, then introduced into the pCRCT plasmid using Golden Gate reaction using BsaI restriction sites [44], and transformed into E. coli cells, and plated on LB + Xgal for blue/white screening and incubated overnight at $37{ }^{\circ} \mathrm{C}$. The bacterial colony with correct plasmid construction was verified by restriction digestion. The constructed CRISPR-Cas9 plasmid was then transformed into YAG114 and YAG118 strains and selected on SC-uracil plates. Colonies were picked and target mutations were first verified using PCR amplification refractory mutation system (ARMS) as described by [45] followed by confirmation via Sanger sequencing. Cas9 plasmid was cured by serially passaging the strain in YPD 3 times and verified by PCR amplification.

\section{Abbreviations \\ ALE: Adaptive laboratory evolution; FPP: Farnesyl pyrophosphate; PCR: Polym- erized chain reaction; SC: Synthetic complete; DCW: Dry cell weight.}

\section{Supplementary Information}

The online version contains supplementary material available at https://doi. org/10.1186/s12934-021-01598-z.

Additional file 1. List of Additional tables and figures.

\section{Acknowledgements}

This work was partially financially supported by NSF CBET-1605347.

\section{Authors' contributions}

AG conducted the experiments, analyzed the data, and contributed to the manuscript. KK analyzed the data and contributed to the manuscript. All authors read and approved the final manuscript.

\section{Funding}

This work was supported by NSF CBET-1605347 (KK).

\section{Availability of data and materials}

The datasets during and/or analysed during the current study available from the corresponding author on reasonable request.

\section{Declarations}

Ethics approval and consent to participate Not applicable.
Consent for publication

Not applicable.

\section{Competing interests}

The authors declare that they have no competing interests.

\section{Author details}

${ }^{1}$ Department of Chemical Engineering, Texas A\&M University, College Station, TX, USA. ${ }^{2}$ Department of Chemical and Materials Engineering, San Jose State University, One Washington Sq, San Jose, CA 95192, USA.

Received: 16 March 2021 Accepted: 20 May 2021

Published online: 27 May 2021

\section{References}

1. Arigoni D, Sagner S, Latzel C, Eisenreich W, Bacher A, Zenk MH. Terpenoid biosynthesis from 1-deoxy-D-xylulose in higher plants by intramolecular skeletal rearrangement. Proc Natl Acad Sci USA. 1997;94(20):10600-5.

2. Asadollahi MA, Maury J, Moller K, Nielsen KF, Schalk M, Clark A, et al. Production of plant sesquiterpenes in Saccharomyces cerevisiae: effect of ERG9 repression on sesquiterpene biosynthesis. Biotechnol Bioeng. 2008;99(3):666-77.

3. Wu W, Liu F, Davis RW. Engineering Escherichia coli for the production of terpene mixture enriched in caryophyllene and caryophyllene alcohol as potential aviation fuel compounds. Metab Eng Commun. 2018;6:13-21.

4. Ignea C, Pontini M, Motawia MS, Maffei ME, Makris AM, Kampranis SC Synthesis of 11-carbon terpenoids in yeast using protein and metabolic engineering. Nat Chem Biol. 2018;14(12):1090-8.

5. Wang C, Liwei M, Park J-B, Jeong S-H, Wei G, Wang Y, et al. Microbial platform for terpenoid production: Escherichia coli and yeast. Front Microbiol. 2018;9:2460.

6. Russo A, Perri M, Cione E, Di Gioia ML, Nardi M, Cristina CM. Biochemical and chemical characterization of Cynara cardunculus L. extract and its potential use as co-adjuvant therapy of chronic myeloid leukemia. J Ethnopharmacol. 2017:202:184-91.

7. Hüsnü K, Başer C, Demirci F. Chemistry of essential oils. In: Berger RG, editor. Flavours and fragrances: chemistry, bioprocessing and sustainability. Berlin: Springer; 2007. p. 43-86.

8. Tundis R, lacopetta D, Sinicropi MS, Bonesi M, Leporini M, Passalacqua NG, et al. Assessment of antioxidant, antitumor and pro-apoptotic effects of Salvia fruticosa Mill. subsp. thomasii (Lacaita) Brullo, Guglielmo, Pavone \& Terrasi (Lamiaceae). Food Chem Toxicol. 2017;106(Pt A):155-64.

9. Dahham SS, Tabana YM, lqbal MA, Ahamed MB, Ezzat MO, Majid AS, et al. The anticancer, antioxidant and antimicrobial properties of the sesquiterpene beta-caryophyllene from the essential oil of Aquilaria crassna. Molecules (Basel, Switzerland). 2015;20(7):11808-29.

10. Reinsvold RE, Jinkerson RE, Radakovits R, Posewitz MC, Basu C. The production of the sesquiterpene beta-caryophyllene in a transgenic strain of the cyanobacterium Synechocystis. J Plant Physiol. 2011;168(8):848-52.

11. Yang J, Nie Q. Engineering Escherichia coli to convert acetic acid to ß-caryophyllene. Microb Cell Fact. 2016;15:74.

12. Alper H, Miyaoku K, Stephanopoulos G. Construction of lycopeneoverproducing E. coli strains by combining systematic and combinatorial gene knockout targets. Nat Biotechnol. 2005;23(5):612-6.

13. de Ruijter JC, Jurgens G, Frey AD. Screening for novel genes of Saccharomyces cerevisiae involved in recombinant antibody production. FEMS Yeast Res. 2017. https://doi.org/10.1093/femsyr/fow104.

14. Prelich G. Gene overexpression: uses, mechanisms, and interpretation. Genetics. 2012;190(3):841-54.

15. Arlt $\mathrm{H}$, Perz A, Ungermann C. An overexpression screen in Saccharomyces cerevisiae identifies novel genes that affect endocytic protein trafficking. Traffic. 2011;12(11):1592-603.

16. Stevenson LF, Kennedy BK, Harlow E. A large-scale overexpression screen in Saccharomyces cerevisiae identifies previously uncharacterized cell cycle genes. Proc Natl Acad Sci. 2001;98(7):3946-51.

17. Portnoy VA, Bezdan D, Zengler K. Adaptive laboratory evolution-harnessing the power of biology for metabolic engineering. Curr Opin Biotechnol. 2011;22(4):590-4. 
18. Reyes LH, Gomez JM, Kao KC. Improving carotenoids production in yeast via adaptive laboratory evolution. Metab Eng. 2014;21:26-33.

19. Promdonkoy P, Mhuantong W, Champreda V, Tanapongpipat S, Runguphan W. Improvement in d-xylose utilization and isobutanol production in S. cerevisiae by adaptive laboratory evolution and rational engineering. J Ind Microbiol Biotechnol. 2020;47(6):497-510.

20. Rugbjerg P, Feist AM, Sommer MOA. Enhanced metabolite productivity of Escherichia coli adapted to glucose M9 minimal medium. Front Bioeng Biotechnol. 2018:6:166.

21. Calleja MA, Vieites JM, Montero-Melendez T, Torres MI, Faus MJ, Gil A, et al. The antioxidant effect of beta-caryophyllene protects rat liver from carbon tetrachloride-induced fibrosis by inhibiting hepatic stellate cell activation. Br J Nutr. 2013;109(3):394-401.

22. Michaelis S. STE6, the yeast a-factor transporter. Semin Cell Biol. 1993;4(1):17-27.

23. Raymond M, Gros P, Whiteway M, Thomas DY. Functional complementation of yeast ste6 by a mammalian multidrug resistance mdr gene. Science (New York, NY). 1992;256(5054):232-4.

24. Ruegg TL, Kim E-M, Simmons BA, Keasling JD, Singer SW, Soon Lee T, et al. An auto-inducible mechanism for ionic liquid resistance in microbial biofuel production. Nat Commun. 2014;5(1):3490.

25. Eng T, Demling P, Herbert RA, Chen Y, Benites V, Martin J, et al. Restoration of biofuel production levels and increased tolerance under ionic liquid stress is enabled by a mutation in the essential Escherichia coli gene cydC. Microb Cell Fact. 2018;17(1):159.

26. Berkower $C$, Michaelis $S$. Mutational analysis of the yeast a-factor transporter STE6, a member of the ATP binding cassette (ABC) protein superfamily. EMBO J. 1991;10(12):3777-85.

27. Walker JE, Saraste M, Runswick MJ, Gay NJ. Distantly related sequences in the alpha- and beta-subunits of ATP synthase, myosin, kinases and other ATP-requiring enzymes and a common nucleotide binding fold. EMBO J. 1982;1(8):945-51.

28. Proff $C$, Kölling R. Functional asymmetry of the two nucleotide binding domains in the ABC transporter Ste6. Mol Gen Genet. 2001;264(6):883-93.

29. Kim JM, Vanguri S, Boeke JD, Gabriel A, Voytas DF. Transposable elements and genome organization: a comprehensive survey of retrotransposons revealed by the complete Saccharomyces cerevisiae genome sequence. Genome Res. 1998;8(5):464-78.

30. Sandmann T, Herrmann JM, Dengjel J, Schwarz H, Spang A. Suppression of coatomer mutants by a new protein family with COPI and COPII binding motifs in Saccharomyces cerevisiae. Mol Biol Cell. 2003;14(8):3097-113.

31. Gresham D, Desai MM, Tucker CM, Jenq HT, Pai DA, Ward A, et al. The repertoire and dynamics of evolutionary adaptations to controlled nutrient-limited environments in yeast. PLoS Genet. 2008;4(12):e1000303.

32. Wilke CM, Adams J. Fitness effects of Ty transposition in Saccharomyces cerevisiae. Genetics. 1992;131(1):31-42.
33. Wallace-Salinas V, Brink DP, Ahrén D, Gorwa-Grauslund MF. Cell peripheryrelated proteins as major genomic targets behind the adaptive evolution of an industrial Saccharomyces cerevisiae strain to combined heat and hydrolysate stress. BMC Genomics. 2015;16(1):514.

34. Fidalgo M, Barrales RR, Ibeas JI, Jimenez J. Adaptive evolution by mutations in the FLO11 gene. Proc Natl Acad Sci USA. 2006;103(30):11228-33.

35. Watanabe J, Uehara K, Mogi Y. Adaptation of the osmotolerant yeast Zygosaccharomyces rouxii to an osmotic environment through copy number amplification of FLO11D. Genetics. 2013;195(2):393-405.

36. Almario MP, Reyes LH, Kao KC. Evolutionary engineering of Saccharomyces cerevisiae for enhanced tolerance to hydrolysates of lignocellulosic biomass. Biotechnol Bioeng. 2013;1 10(10):2616-23.

37. Brachmann CB, Davies A, Cost GJ, Caputo E, Li J, Hieter P, et al. Designer deletion strains derived from Saccharomyces cerevisiae S288C: a useful set of strains and plasmids for PCR-mediated gene disruption and other applications. Yeast. 1998;14(2):115-32.

38. Lee ME, DeLoache WC, Cervantes B, Dueber JE. A highly characterized yeast toolkit for modular, multipart assembly. ACS Synth Biol. 2015;4(9):975-86.

39. Rodriguez S, Kirby J, Denby CM, Keasling JD. Production and quantification of sesquiterpenes in Saccharomyces cerevisiae, including extraction, detection and quantification of terpene products and key related metabolites. Nat Protoc. 2014;9(8):1980-96.

40. Sousa JP, Brancalion AP, Souza AB, Turatti IC, Ambrosio SR, Furtado NA, et al. Validation of a gas chromatographic method to quantify sesquiterpenes in copaiba oils. J Pharm Biomed Anal. 2011;54(4):653-9.

41. Kahm M, Hasenbrink G, Lichtenberg-Fraté H, Ludwig J, Kschischo M. grofit: fitting biological growth curves with R. J Stat Softw. 2010. https:// doi.org/10.18637/jss.v033.i07.

42. Deatherage DE, Barrick JE. Identification of mutations in laboratoryevolved microbes from next-generation sequencing data using breseq. Methods Mol Biol (Clifton, NJ). 2014;1151:165-88.

43. Bao Z, Xiao H, Liang J, Zhang L, Xiong X, Sun N, et al. Homology-integrated CRISPR-Cas (HI-CRISPR) system for one-step multigene disruption in Saccharomyces cerevisiae. ACS Synth Biol. 2015;4(5):585-94.

44. Engler C, Kandzia R, Marillonnet S. A one pot, one step, precision cloning method with high throughput capability. PLoS ONE. 2008;3(11):e3647.

45. Little S. Amplification-refractory mutation system (ARMS) analysis of point mutations. In: Current protocols in human genetics. 2001; Chapter 9:Unit 9.8.

\section{Publisher's Note}

Springer Nature remains neutral with regard to jurisdictional claims in published maps and institutional affiliations.
Ready to submit your research? Choose BMC and benefit from:

- fast, convenient online submission

- thorough peer review by experienced researchers in your field

- rapid publication on acceptance

- support for research data, including large and complex data types

- gold Open Access which fosters wider collaboration and increased citations

- maximum visibility for your research: over $100 \mathrm{M}$ website views per year

At BMC, research is always in progress.

Learn more biomedcentral.com/submissions 